\title{
Superior mesenteric artery syndrome
}

\author{
PETER A. JONES \\ F.R.C.S.
}

C. WASTELL

M.S., F.R.C.S.

Westminster Hospital, London SWIP 2AP

\begin{abstract}
Summary
Superior mesenteric artery syndrome (SMAS) was diagnosed in a 16-year-old boy who presented with a short history of repeated vomiting associated with weight loss to the point of collapse. After resuscitation and investigation, he was treated surgically and subsequently recovered well. The diagnostic difficulties and possible pathogenesis of SMAS are discussed and the literature reviewed.
\end{abstract}

KEY WORDS: duodenum, obstruction, gastrointestinal.

\section{Case report}

A 16-year-old apprentice motor mechanic, was admitted as an emergency unable to walk without assistance because of extreme weakness. He had been vomiting for 8 weeks, initially 2-3 times weekly, but increasing to 3-4 times daily during the 2 weeks before admission. The vomitus consisted of undigested food eaten over the past day or two. During this 8 week period, he had lost $19 \mathrm{~kg}$ in weight.

On examination, he was extremely thin and weak, but there were no other abnormal physical signs. Investigations showed a moderate hypokalaemic alkalosis and a blood urea of $22.8 \mathrm{mmol} /$ litre. The barium meal showed obstruction of the third part of the duodenum with proximal dilatation (Fig. 1).

The differential diagnosis was thought to include superior mesenteric artery syndrome (SMAS), small bowel lymphoma, external compression from an abnormal band, annular pancreas or intraduodenal diverticulum. After adequate rehydration and correction of the metabolic disturbances, a laparotomy was performed. The first and second parts of the duodenum were dilated and hypertrophied but there was no other abnormality. Their dilatation came to an abrupt end where the superior mesenteric vessels crossed the third part of the duodenum. Duodenotomy excluded an intraduodenal abnormality. It was concluded that this was a case of superior mesenteric artery syndrome and a short looped, retrocolic duodenojejunostomy was fashioned (Fig. 2). The

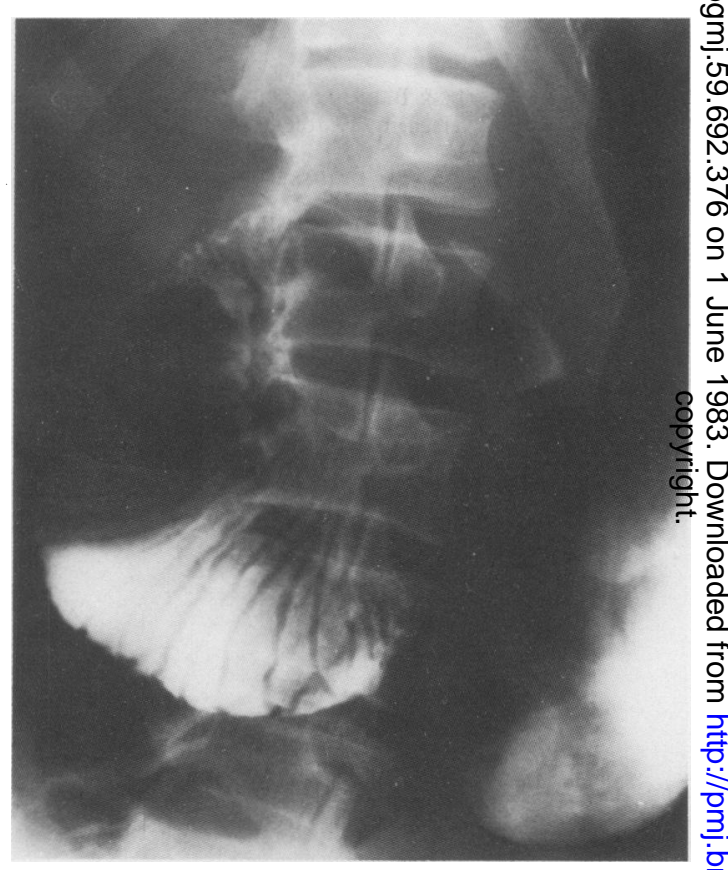

FIG. 1. Barium meal showing obstruction of the third part of the duodenum with proximal dilatation.

patient made an uneventful recovery and gained $18 \stackrel{\circ}{\stackrel{\circ}{~}}$ $\mathrm{kg}$ in the first 5 postoperative weeks. One year later, he remains asymptomatic.

\section{Discussion}

Historically, Von Rokitansky (1861) was the first to 0 describe duodenal dilatation and obstruction due to

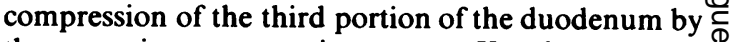
the superior mesenteric artery. Kundrat (1891), 保 Albrecht (1899) and Robinson (1900) added further 0 cases and in 1907, Bloodgood suggested that this potentially life-threatening condition could be surgi- $\overrightarrow{\mathbb{D}}$ cally treated by a duodenojejunostomy. This proce- $\frac{\overparen{\Phi}}{\Phi}$ 


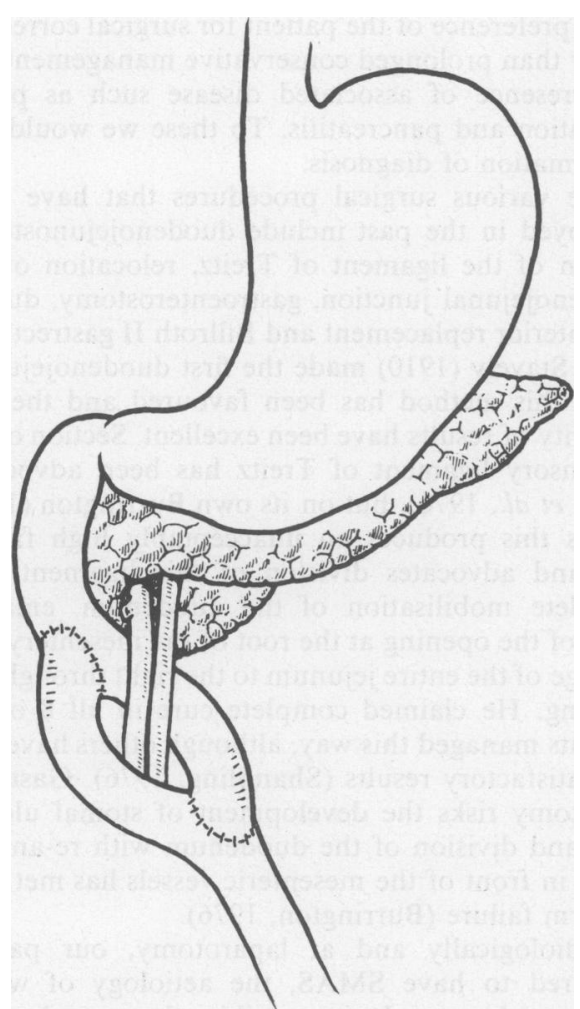

FIG. 2. Diagram of operative procedure performed: retrocolic duodenojejunostomy with a very short loop.

dure was first performed successfully by Stavely the following year (Stavely, 1910).

Vascular compression of the duodenum has, during the years, received other names, such as arteriomesenteric duodenal compression syndrome and the cast syndrome. Wilkie (1921) called it 'chronic duodenal ileus'. He subsequently reported 75 cases (Wilkie, 1927) and although his name has been applied to the syndrome it is still usually known as the 'superior mesenteric artery syndrome'.

The popular, but often disputed, pathogenetic principle is that the duodenum, either the third or fourth part, is compressed between the aorta and vertebral column posteriorly and the superior mesenteric vessels anteriorly (Fig. 3). In a normal person, the mass of fat and lymphatic tissue around the origin of the superior mesenteric artery is sufficient to protect the duodenum from compression as it crosses the spine and aorta. However, an increased lordosis, a short mesentery, sudden weight loss or a rapid growth in height will narrow the angle and, in susceptible patients, this will cause partial, or even complete occlusion of the duodenum. Other factors implicated include a high attachment of the ligament

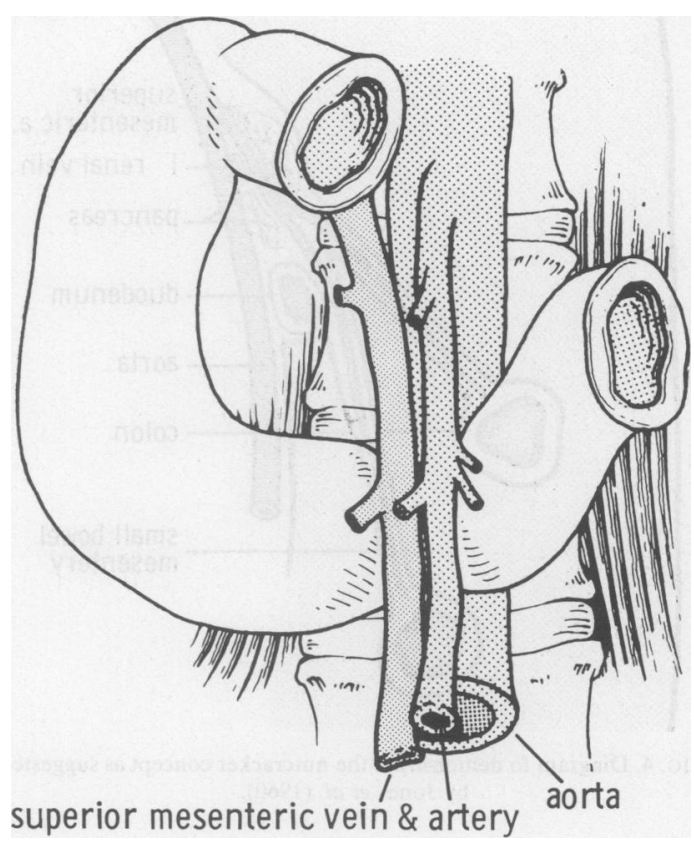

Fig. 3. Diagram of the anatomy of the third part of the duodenum and its relationship to the vertebrae and superior mesenteric vessels.

of Treitz and external pressure such as application of a body cast in the treatment of scoliosis or vertebral fracture.

Arteriographic studies in patients with extensive burns have confirmed that the angle formed by the superior mesenteric artery may be decreased to as little as 15 degrees in patients who have lost weight rapidly from debilitating injuries (Wayne and Burrington, 1972). Furthermore, simultaneous angiography and barium studies have shown that the site of duodenal compression corresponds closely to the position of the superior mesenteric artery (Wayne, Miller and Eiseman, 1971), and Jones et al. (1960) likened this arrangement to a nutcracker (Fig. 4). Burrington (1976) thought this concept was too simplistic and suggested that compression resulted from the attachment of the small bowel mesentery to the retro-peritoneal tissue and the peritoneal reflection that forms the ligament of Treitz. He highlighted the difficulty of distinguishing SMAS from psychogenic vomiting by describing a girl in whom anorexia nervosa had been diagnosed. Force feeding was complicated by gastric perforation, but after correction of her duodenal obstruction and replacement of the resected stomach with colon, she regained her weight willingly.

To date just over 300 well-defined cases have been described in the literature. The condition affects young adults and men to women in the proportion of 


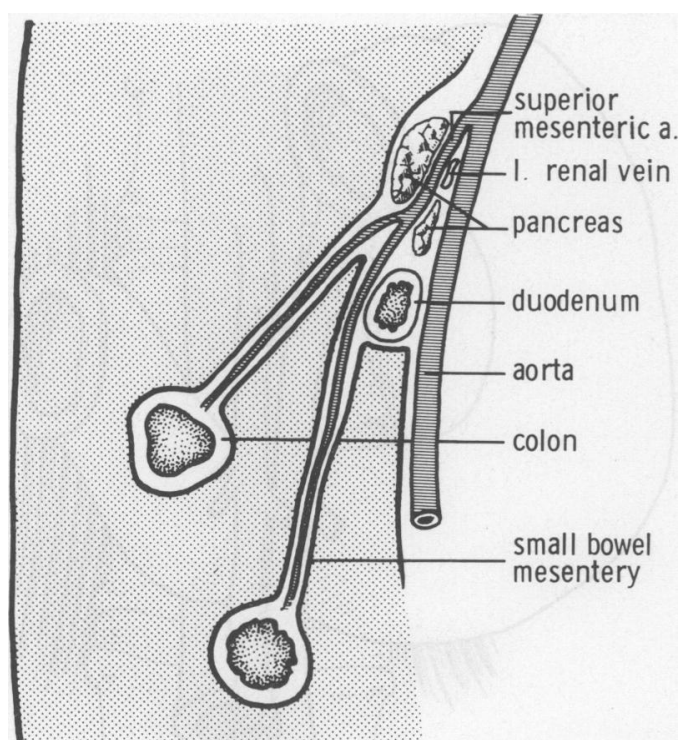

FIG. 4. Diagram to demonstrate the nutcracker concept as suggested by Jones et al. (1960).

2 to 3 . The chief symptoms of the superior mesentery artery syndrome are vomiting and epigastric pain. The disease is progressive with vomiting of increasing severity and associated weight loss. In a review by Akin, Gray and Skandalakis (1976), half the patients had suffered for 6 months or less, although there is a more chronic form and one patient is reported as having the condition for 23 years.

Obstruction to the passage of barium in the third portion of the duodenum with a straight line cut-off and proximal dilatation are the most important diagnostic criteria (Anderson et al., 1973). Reverse peristalsis in the dilated segment proximal to the obstruction may also be seen. Hayes manoeuvre is the demonstration of the disappearance of these radiological features in the knee-chest position on cinefluoroscopy (Lundell and Thulin, 1980), and interestingly some relief of symptoms may be achieved in this way.

Most authors believe a trial of medical management should precede surgical intervention. Treatment consists of frequent meals of pureed or blenderized food diluted to the consistency of soup. By taking 180-240 $\mathrm{ml}$ every $4 \mathrm{hr}$ and then lying on the left side, prone, or in the knee-chest position the patient may eventually gain weight. Intravenous alimentation has also been used. Review of the published cases shows that over $75 \%$ eventually come to surgery, perhaps partly to establish the diagnosis. The indications for surgical intervention were summarized by Berner and Sherman (1963) and include failure of a strict medical regime to alleviate symp- toms, preference of the patient for surgical correction 3 rather than prolonged conservative management and $₫$ the presence of associated disease such as peptic $c$ ulceration and pancreatitis. To these we would add $\overrightarrow{\vec{F}}$ confirmation of diagnosis.

The various surgical procedures that have been employed in the past include duodenojejunostomy, section of the ligament of Treitz, relocation of the $\overrightarrow{ }$ duodenojejunal junction, gastroenterostomy, duode- $\Omega$ nal anterior replacement and Billroth II gastrectomy. ळ Since Stavely (1910) made the first duodenojejunos- $\overrightarrow{0}$ tomy, this method has been favoured and the vast majority of results have been excellent. Section of the $\vec{\omega}$ suspensory ligament of Treitz has been advocated (Akin et al., 1976), but on its own Burrington (1976) claims this produces an unacceptably high failure rate and advocates division of this ligament with complete mobilisation of the duodenum, enlarge- is ment of the opening at the root of the mesentery and $N$ passage of the entire jejunum to the right through this opening. He claimed complete cure in all 8 of his patients managed this way, although others have had less satisfactory results (Shandling, 1976). Gastroje- $\vec{c}$ junostomy risks the development of stomal ulceration, and division of the duodenum with re-anastomosis in front of the mesenteric vessels has met with $\overrightarrow{0}$ uniform failure (Burrington, 1976).

Radiologically and at laparotomy, our patien appeared to have SMAS, the aetiology of whick remains obscure. It is possible that psychogenic vomiting led to sudden weight loss, which combined with a growth spurt, precipitated SMAS. Certainly without surgery, it is difficult to see how the patient would have survived and duodenojejunostomy has worked well.

\section{References}

AKIN, J.T., GraY, S.W. \& Skandalakis, J.E. (1976) Vascular compression of the duodenum: presentation of ten cases and review of the literature. Surgery, 79, 515 .

ALBRECHT, P.A. (1899) Uber arterio-mesenterialen dormverschluss an der duodeno-jejunal-grenze, und seine ursachlige berziehung zur magenweiterung. Virchows Archiv für pathologische Anatomie und Physiologie, 156, 285.

ANDERSON, W.C., Vivit, R., KirSh, I.E. \& GReEnleE, H.B. (1973) Arteriomesenteric duodenal compression syndrome. American Journal of Surgery, 125, 681.

BARNER, H.B. \& SHERMAN, C.D., Jr. (1963) Vascular compression of the duodenum. International Abstracts of Surgery, 117, 103.

BLOODGOOD, J.C. (1907) Acute dilatation of the stomach; gastro- N mesenteric ileus. Annals of Surgery, 46, 736.

BURRINGTON, J.D. (1976) Superior mesenteric artery syndrome in children. American Journal of Diseases of Children, 130, 1367.

Jones, S.A., CARTer, R., Smith, L.L. \& Joergenson, E.J. (1960) Arteriomesenteric duodenal compression. American Journal of $\mathscr{S}$ Surgery. 100, 262.

KUNISRAT, I. (1891) Ueber eine seltene form der inneren incarceration. Wiener medizinische Wochenschrift. 41, 352.

LUNIDIII. L. \& THUI.IN, A. (1980) Wilkie's syndrome a rarity? British Journal of Surgery. 67, 604. 
ROBINSON, B. (1900) Dilatation of the stomach from pressure of the superior mesenteric artery, vein and nerve on the transverse segment of the duodenum. Cincinnati Lancet-Clinic, 45, 557.

ROKITANSKY, C., VON. (1861) Lehrbuch der patologische Anatomie (Ed. I. Vienna), p. 187. Braumuller.

SHANDLING, B. (1976) The so-called superior mesenteric artery syndrome. American Journal of Diseases of Children, 130, 1371.

Staveley, A.L. (1910) Chronic gastromesenteric ileus. Surgery, Gynecology and Obstetrics, 11, 288.
WAYNE, E.R. \& BURRINGTON, J.D. (1972) Duodenal obstruction by the superior mesenteric artery in children. Surgery, 72, 762.

Wayne, E.R., Miller, R.E. \& Eiseman, B. (1971) Duodenal obstruction by the superior mesenteric artery in bedridden combat casualties. Annals of Surgery, 174, 339.

WILKIE, D.P.D. (1921) Chronic duodenal ileus. British Journal of Surgery, 9, 204.

WILKIE, D.P.D. (1927) Chronic duodenal ileus. American Journal of the Medical Sciences, 173, 643.

(Accepted 19 October 1982) 